\title{
FERMENTAÇÃO DE PETIT SUISSE OBTIDO COM RETENÇÃO DE SORO E ADIÇÃO DE LACTASE E REDUÇÃO DA ADIÇÃO DE AÇÚCARES VERSUS À FORMULAÇÃO TRADICIONAL
}

\section{Fermentation of Petit Suisse obtained with whey retention, lactase addition and less amount of added sugars versus traditional formulation}

Isis Rodrigues Toledo Renhe ${ }^{*}$, Alisson Borges de Souza ${ }^{2}$, Júlia d'Almeida Francisquini ${ }^{3}$, Danielle Braga Chelini Pereira ${ }^{4}$, Antônio Fernandes de Carvalho ${ }^{2}$, Ítalo Tuler Perrone ${ }^{5}$

\section{RESUMO}

As atuais tendências de mercado apontam para produtos livres ou com baixo teor de lactose. Além disso, questões econômicas e ecológicas levam a adaptação das tradicionais tecnologias por outras que permitam modificações nos processos que atendam à nova realidade sem, contudo, perder em qualidade. Entretanto, o desenvolvimento de novas tecnologias exigem estudos que possam otimizá-las de modo a atender às necessidades da indústria e do consumidor. $\mathrm{O}$ presente trabalho teve como objetivo avaliar os impactos do uso de lactase e da redução dos teores de sacarose adicionado na fermentação de Petit Suisse pelo método da retenção de soro. A utilização de lactase na produção do queijo Petit Suisse reduziu em duas horas o tempo de fermentação em relação à formulação tradicional nas condições experimentais empregadas neste trabalho. O perfil da fermentação diferiu entre as formulações estudadas e foram estabelecidas relações matemáticas entre a variação do $\mathrm{pH}$ e o tempo.

1 Empresa de Pesquisa Agropecuária de Minas Gerais, Instituto de Laticínios Cândido Tostes (EPAMIG/ILCT), Rua Tenente Luiz de Freitas, 116, 36045-560, Juiz de Fora, MG, Brasil. E-mail: isis@epamig.br

2 FMC Health and Nutrition, Campinas, SP, Brasil.

3 Universidade Federal de Viçosa, Departamento de Tecnologia de Alimentos, Viçosa, MG, Brasil.

4 Laticínios Bela Vista, Bela Vista de Goiás, GO, Brasil.

5 Universidade Federal de Juiz de Fora, Departamento de Ciências Farmacêuticas, Juiz de Fora, MG, Brasil.

* Autor para correspondência. 
Palavras-chave: queijo; fermentação; desenvolvimento de novos produtos; intolerância a lactose.

\begin{abstract}
Current market trends indicate lactose free or low lactose products. In addition, economic and ecological issues lead to the adaptation of traditional technologies to other ones that allow process modification to meet the new reality without losing quality. However, the development of new technologies demands studies to optimize them in order to meet industries and consumers necessities. The present work aimed to evaluate the impacts of the use of lactase and the reduction of sugar addition in the fermentation of Petit Suisse made with the method of whey retention. The applying of lactase during Petit Suisse production allows the reduction in fermentation time in two hours according to the experimental protocol of the experiment. The fermentation profile differs among treatments and mathematical relations between $\mathrm{pH}$ and time were established.
\end{abstract}

Keywords: cheese; fermentation; development of new products; lactose intolerance.

\section{INTRODUÇ̃̃O}

A fermentação ácido lática é realizada há anos na indústria de alimentos, com destaque para a produção de lácteos tradicionais como o iogurte. As consequências da fermentação incluem a mudança na microestrutura das proteínas do leite, caracterizam a textura e o sabor de lácteos fermentados e impactam favoravelmente nos atributos nutricionais dos produtos (SAUBADE et al., 2017).

A produção do queijo Petit Suisse caracteriza-se classicamente pela fermentação do tipo mesofílica sem a adição de lactase e atualmente alternativas tecnológicas tem sido buscadas visando a redução da produção de soro ácido (tecnologia por formulação), incorporação de novos ingredientes, aditivos e adoçantes (ESMERINO et al., 2013; PEREIRA et al., 2016).

A produção de lácteos com teores reduzidos em lactose estabeleceu-se nos últimos anos no país e diversas pesquisas sobre o desenvolvimento de novos produtos baseados na redução da lactose foram publicadas
(MÄKINEN et al., 2015; OHLSSON et al., 2017; TORRES et al., 2017).

$\mathrm{Na}$ produção de queijos boa parte da lactose é eliminada na dessoragem e não chega a se tornar um problema para as pessoas intolerantes. Entretanto, a produção de Petit Suisse por formulação, com retenção de soro, faz com que a lactose volte a se tornar um problema, uma vez que a fermentação por bactérias láticas reduz apenas parcialmente os teores de lactose no produto final (DEKKER, DAAMEN, 2011), justificando o uso da lactase. O objetivo deste trabalho foi avaliar os impactos do uso de lactase na fermentação do Petit Suisse pelo método da retenção de soro.

\section{MATERIAL E MÉTODOS}

A produção dos queijos Petit Suisse foi realizada no laboratório de desenvolvimento da empresa GemacomTech e as análises físico-químicas e sensoriais foram realizadas no Laboratório de Pesquisa de Leite e Derivados do Instituto de Laticínios Cândido Tostes. 


\section{Formulação dos Produtos}

Foi produzido um Petit Suisse controle segundo a tecnologia de formulação, com ausência de dessoragem, que tem sido amplamente difundida no mercado. Nesse processo o leite $\left(65,42 \% \mathrm{~m} \cdot \mathrm{m}^{-1}\right)$ é adicionado de $8,5 \% \mathrm{~m} \cdot \mathrm{m}^{-1}$ de creme (36\% de gordura), sacarose $\left(15 \% \mathrm{~m} \cdot \mathrm{m}^{-1}\right)$, agentes de corpo compostos por hidrocolóides (Tecgem AA772, $6 \% \mathrm{~m} \cdot \mathrm{m}^{-1}$ ), polpa (Tecgem AA 21.220, $\left.5 \% \mathrm{~m} \cdot \mathrm{m}^{-1}\right)$ e citrato de sódio $\left(0,08 \% \mathrm{~m} \cdot \mathrm{m}^{-1}\right)$. Estes ingredientes foram misturados e aquecidos até temperatura de $90{ }^{\circ} \mathrm{C}$ por 3 minutos, sendo homogeneizado a pressão de 200 bar quando atingida a temperatura de $50{ }^{\circ} \mathrm{C}$. O produto formulado foi fermentado com fermento termofílico a $38 \pm 2{ }^{\circ} \mathrm{C}$ (MOS $062 \mathrm{E}$ - Sacco), composto pelos microrganismos Lactococcus lactis subsp. lactis, Lactococcus lactis subsp. cremoris e Streptococcus thermophilus, até $\mathrm{pH}$ próximo de 4,6. Após atingir o final da fermentação, o produto foi resfriado e texturizado em homogeneizador a pressão de 50 bar, para atingir a textura final desejada no produto.

No segundo tratamento foi realizada a mesma fabricação descrita anteriormente, diferenciando-se pela adição da enzima lactase (SternEzym 21.901L) no início da fermentação, juntamente com o fermento. Foi empregada a dose recomendada pelo fabri-

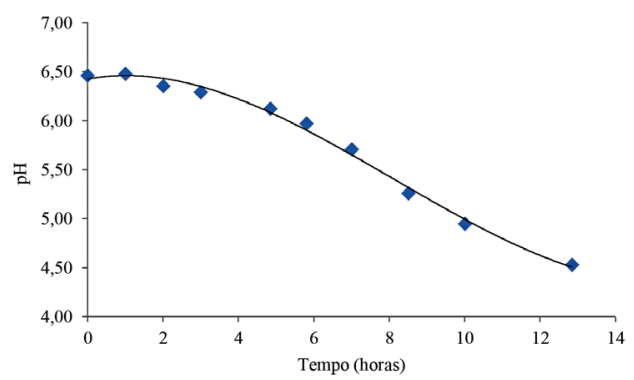

Figura 1 - Variação do $\mathrm{pH}$ versus o tempo para o Tratamento $(\mathrm{n}=3)$ cante, a concentração de $0,05 \%$ para cada 1 $\mathrm{g}$ de lactose. Foi feita ainda a diminuição de sacarose em $20 \%$ com relação ao controle.

\section{Análises físico-químicas}

Os produtos foram fabricados em três repetições. A avaliação da fermentação dos produtos foi realizada pela medição do $\mathrm{pH}$ durante 14 horas e/ou até $\mathrm{pH}$ próximo de 4,6. O pH foi medido com medidor de $\mathrm{pH}$ (Pereira et al., 2001). Além da avaliação do $\mathrm{pH}$ versus tempo, foi analisado a taxa de variação infinitesimal do $\mathrm{pH}$ versus o tempo de fermentação $(\mathrm{dpH} / \mathrm{dT})$. Os resultados foram analisados pelo programa estatístico Origin 5.0 com ajuste do modelo de equação da reta.

\section{RESULTADOS E DISCUSSÃO}

Nas Figuras 1 e 2 são apresentadas as variações do $\mathrm{pH}$ durante o tempo de fermentação para o Tratamento (adição de lactase) e para o Controle, respectivamente. O tempo de fermentação foi superior ao indicado pelos fabricantes do fermento, por se tratar de produto formulado, em que a fermentação foi conduzida com todos os constituintes do produto final. Em condições normais, o tempo esperado para o fermento utilizado é de 4 a 8 horas. No experimento, a fermentação teve

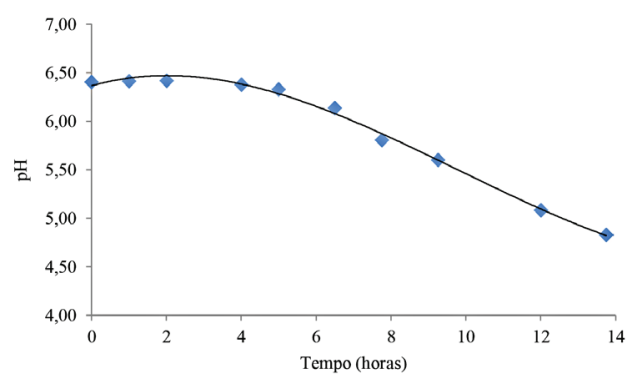

Figura 2 - Variação do $\mathrm{pH}$ versus o tempo para o Controle $(\mathrm{n}=3)$ 
duração de 11 a 15 horas, com tempo sempre superior para o tratamento controle. Tal aumento também já era esperado, uma vez que o fermento apresenta aumento considerável no tempo de fermentação para produtos com teores de açúcar superiores a 15\%. Ainda segundo os fornecedores do fermento, esses teores de sacarose também levam a menor viscosidade no produto final.

Na Figura 1 os dados da variação do $\mathrm{pH}$ durante o tempo de fermentação para o Tratamento foram ajustados a um modelo de terceiro grau. O modelo é apresentado na equação 1 .

$p H=0,0015 T^{3}-0,036 T^{2}+0,069 T+$ $6,43\left(R^{2}=0,9937\right)$

Na Figura 2 os dados da variação do $\mathrm{pH}$ durante o tempo de fermentação para o Controle foram ajustados a um modelo de terceiro grau. O modelo é apresentado na equação 2 .

$p H=0,001 T^{3}-0,0303 T^{2}+0,1092 T+$ $6,37\left(R^{2}=0,9953\right)$

As equações 1 e 2 podem ser empregadas industrialmente, dentro das condições de fermentação padronizadas neste trabalho, para a previsão do tempo de fermentação necessário para se atingir o $\mathrm{pH}$ desejado pela indústria. Também podem servir de referência para o controle do processo no tocante a variação do $\mathrm{pH}$ com o tempo de fermentação, desta forma, valores de $\mathrm{pH}$ determinados durante a produção industrial muito distintos dos calculados pelas equações podem indicar algum problema na fermentação, possibilitando a adoção de uma medida corretiva de controle durante esta etapa da fabricação.

Ao calcularmos a derivada a primeira (taxa de variação infinitesimal) das equações 1 e 2 determinamos duas equações que representam fisicamente a velocidade de variação do $\mathrm{pH}$ com o tempo respectivamente para o tratamento e para o controle. Estas derivadas são apresentadas na Figura 3 e pelas Equações 3 e 4.

$d p H / d T=0,0045 T^{2}-0,072 T+0,069$

$d p H / d T=0,003 T^{2}-0,0606 T+0,1092$

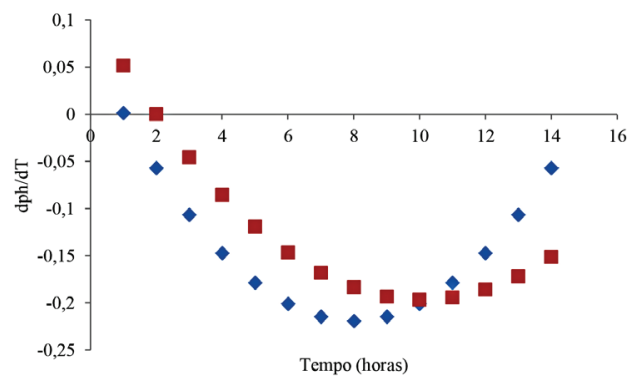

Figura 3 - Taxa de variação infinitesimal do $\mathrm{pH}$ versus o Tempo de fermentação $(\mathrm{dpH} / \mathrm{dT})$

Pela análise da Figura 3, observa-se que a velocidade com que o $\mathrm{pH}$ diminui é maior para o Tratamento quando comparada ao Controle até o tempo de aproximadamente 10 horas de fermentação. Com 10 horas de fermentação, as velocidade de variação do $\mathrm{pH}$ com o tempo são praticamente iguais e com magnitude de aproximadamente $-0,20$ unidades de $\mathrm{pH}$ por hora. A partir deste ponto, o Controle passa a ter uma maior velocidade de diminuição do pH, comportamento que é mantido até o tempo final da fermentação (14 horas). O fato da velocidade de diminuição do $\mathrm{pH}$ ser maior nas primeiras 10 horas para o Tratamento pode ser atribuída a hidrólise inicial da lactose, uma vez que nesta condição os microrganismos possuem imediatamente à sua disposição moléculas de glicose e de galactose, não necessitando da fase inicial do processo fermentativo que consiste da hidrólise da molécula de lactose. 
O fermento utilizado é constituído dos microrganismos Lactococcus lactis subsp. lactis, Lactococcus lactis subsp. cremoris e Streptococcus thermophilus, todos homofermentadores de lactose. O microrganismo Streptococcus thermophilusse destaca dos demais por ser termófilo e galactose negativo. Ele tem sido incluído em culturas tradicionalmente mesofílicas como adjunct starters para melhorar o aroma do produto fermentado bem como acelerar a produção de ácido lático.

Atingida 10 horas de fermentação, o produto Controle apresenta $\mathrm{pH}$ próximo de 5,5 , enquanto que o Tratamento tem $\mathrm{pH}$ em torno de 5,0. De acordo com Walstra et al. (2006), em geral, os microrganismos presentes no fermentos para queijos tem seu crescimento inibido ou diminuído em $\mathrm{pH}$ próximo de 5,7. Os valores específicos dependem da cepa e os fenômenos envolvidos não são completamente conhecidos. Apesar da paralisação do crescimento, a fermentação e a acidificação continuam.

Acredita-se que a inversão da taxa de diminuição do $\mathrm{pH}$ entre o produto Tratamento e o Controle é função da combinação desses fatores. Num primeiro momento tem-se uma produção de acidez elevada em função da combinação da alta acidificação do microrganismo termófilo e da presença da lactase, que converte a lactose em glicose e galactose. Esses dois açúcares por sua vez podem ser fermentados em conjunto e simultaneamente pelas bactérias do gênero Lactococcus lactis (TAMIME, ROBINSON, 1985). Dessa forma, a segunda fase da fermentação é atingida mais rapidamente pelo produto Tratamento.

Em reações enzimáticas, a formação de produtos pode atuar como inibidor do processo enzimático, desta forma, a galactose e a glicose podem atuar como inibidores da ação da lactase. (DEKKER, DAAMEN, 2011). Por isso, devido ao efeito inibitório da formação de produtos, a lactase gradativamente deixa de ter efeito indireto na acidificação. $\mathrm{Da}$ mesma forma, o abaixamento do $\mathrm{pH}$ leva ao início da inibição do crescimento das cepas do fermento. A temperatura de fermentação $\left(38{ }^{\circ} \mathrm{C}\right)$ também favorece o microrganismo termófilo (Streptococcus thermophilus) que, por ser galactose negativo, passa a ter menos substrato para fermentação em função do consumo de glicose por todos os microrganismos. Assim o desenvolvimento da acidez passa a ser em função da atuação das cepas mesofílicas, que não estão no seu ótimo de desenvolvimento.

Apesar de toda essa inibição e da diminuição da acidificação no produto Controle, essa desaceleração não é significativa em termos de produção, pois a redução da diminuição do $\mathrm{pH}$ acontece próxima ao $\mathrm{pH}$ final do produto não afetando o tempo final de fabricação. Ao contrário, o Petit Suisse da formulação Tratamento apresentou redução média no tempo de fermentação de 2 horas.

\section{CONCLUSÕES}

A utilização de lactase na produção do queijo Petit Suisse reduziu em duas horas o tempo de fermentação em relação à formulação tradicional nas condições experimentais empregadas neste trabalho. O perfil da fermentação diferiu entre as formulações estudadas e foram estabelecidas relações matemáticas entre a variação do $\mathrm{pH}$ e o tempo, podendo ser empregadas para previsões tecnológicas da etapa de fermentação.

\section{AGRADECIMENTOS}

À FAPEMIG pelo financiamento do projeto e bolsas concedidas. Às empresas Gemacom Tech, Sacco do Brasil e Stern Ingredientes pelo fornecimento das amostras para a elaboração dos produtos. 


\section{REFERÊNCIAS}

DEKKER, P. J. T.; DAAMEN, C. B. G. $\beta$-DGalactosidase. In: FUQUAY, J. W.; FOX, P. F.; McSWEENEY, P. L. H. Encyclopedia of Dairy Sciences. 2a ed. London: Elsevier, 2011. p. 276-283.

ESMERINO, E. A. et al. The influence of sweeteners in probiotic Petit Suisse cheese in concentrations equivalent to that of sucrose. Journal of Dairy Science, v. 96, n. 9, p. 5512-5521, 2013.

MÄKINEN, O. E. et al. Physicochemical and acid gelation properties of commercial UHT-treated plant-based milk substitutes and lactose free bovine milk. Food chemistry, v. 168 , p. $630-638,2015$.

OHLSSON, J. A. et al. Lactose, glucose and galactose content in milk, fermented milk and lactose-free milk products. International Dairy Journal, v. 73, p. 151-154, 2017.

PEREIRA, D. B. C.; SILVA, P. H. F. da; JÚNIOR, L. C. G. C.; OLIVEIRA, L. L.de.
Físico-química do leite e derivados - métodos analíticos. $2^{\mathrm{a}}$ ed. Juiz de Fora: Templo, 2001. 234 p.

PEREIRA, E. P. R. et al. Effect of incorporation of antioxidants on the chemical, rheological, and sensory properties of probiotic petit suisse cheese. Journal of Dairy Science, v. 99, n. 3, p. 1762-1772, 2016.

SAUBADE, F. et al. Lactic acid fermentation as a tool for increasing the folate content of foods. Critical reviews in food science and nutrition, v. 57, n. 18, p. 3894-3910, 2017.

TAMIME, A. Y.; ROBINSON, R. K. Yoghurth: Science \& Technology. $1^{\mathrm{a}}$ ed. Oxford: Pergamon, 1985. 431 p.

TORRES, J. K. F. et al. Technological aspects of lactose-hydrolyzed milk powder. Food Research International, v. 101, p. 45-53, 2017.

WALSTRA, P.; GEURTS, T. J.; WALSTRA, P.; WOUTERS, J. T. Dairy Science and Technology. Boca Raton: CRC press, 2006. 808 p. 